\title{
On-Chip Time Measurement Architecture with Femtosecond Timing Resolution
}

\author{
Matthew Collins and Bashir M. Al-Hashimi \\ School of Electronics and Computer Science, University of Southampton, Highfield, \\ Southampton, S017 1BJ, UK \\ Email:mc03r@ecs.soton.ac.uk,bmah@ecs.soton.ac.uk
}

\begin{abstract}
This paper presents a new on-chip time measurement architecture which is based on the Timeto-Digital Conversion (TDC) method that is capable of achieving a timing resolution of tens of femtoseconds without the use of external automatic test equipment (ATE). This is the highest temporal resolution that has been reported to-date and is achieved by the use of the homodyne technique. The proposed architecture has been designed using a $0.12 \mu \mathrm{m}$ CMOS process and simulation results based on foundry transistor models indicates that it is possible to achieve a timing resolution of $40 \mathrm{fs}$. The time measurement architecture is standalone and occupies a small silicon area, $150 \mu \mathrm{m}$ by $180 \mu \mathrm{m}$, making it attractive for high resolution on-chip time measurement.
\end{abstract}

\section{Introduction}

High speed communications has been one of the key driving forces of the electronics industry. The ITRS'05 [1] predicts that on-chip clock speeds will increase into the tens of Gigahertz range which will require time measurement architectures with timing resolutions of tens of femtoseconds. Currently, timing characteristics of VLSI devices are performed using automatic test equipment (ATE). Such testers are able of achieving accurate timing measurements, however they are expensive. Furthermore, the increased integration and performance of VLSI devices due to technology scaling has produced limitations in traditional timing performance test methods. For example, bandwidth and additional timing skew brought about by the increase of electrical distance between the tester and DUT [2]. A solution to address these problems involves placing a single high resolution time measurement architecture directly onto the same device being tested [3]. The use of on-chip time-todigital converters (TDC) is the most effective method for achieving high resolution required for measuring high speed timing parameters. Current techniques for implementing on-chip time-to-digital converters (TDC) have already been proposed. For example, the counter based technique [4], the vernier oscillator [5], time-tovoltage converter techniques [6, 7], Pulse Shrinking [8] and flash based TDC techniques [2]. Table.1 gives a summary of the timing resolution achieved using the various TDC techniques reported to-date. As can be seen, higher timing resolution is achieved with the application of the vernier and flash based TDC techniques as apposed to counter based, pulse shrinking and time-to-voltage techniques. Although the resolutions of the above techniques will undoubtedly increase with technology, this may come at a cost.

Table 1. Recent work on Time-Measurement

\begin{tabular}{lc}
\hline \multicolumn{1}{c}{ Time Measurement Architecture } & Resolution \\
\hline Vernier delay line [9] & $5 \mathrm{ps}$ \\
Flash [2] & $5 \mathrm{ps}$ \\
Time-to-Voltage Conversion [6] & $14 \mathrm{ps}$ \\
Vernier Oscillator [5] & $18.5 \mathrm{ps}$ \\
Interpolator [10] & $30 \mathrm{ps}$ \\
Pulse Shrinking [8] & $57.3 \mathrm{ps}$ \\
Programmable Time-to-Voltage & $103 \mathrm{ps}$ \\
Conversion [7] & \\
\hline
\end{tabular}

Two recent attempts at increasing the temporal resolution of time measurement architectures have been proposed by incorporating time amplifiers that precede the TDC in order to improve the overall timing resolution $[11,12]$. This is achieved by amplifying the time interval into a timing range that the TDC is able to process. However, these techniques are not capable of achieving the required timing resolution since they only provide resolution of hundreds of femtoseconds.

This paper proposes a new architecture capable of producing tens of femtosecond timing resolution and is organised as follows. In section 2, we introduce the principles of the Homodyne technique for time measurement applications and also describe the proposed time measurement architecture. Section 3 describes the importance of analogue-to-digital conversion $(\mathrm{ADC})$ in time measurement architectures and describes the implementation of the $\Delta \Sigma$ ADC. Section 4 shows simulation results using SPECTRE models for a $0.12 \mu \mathrm{m}$ CMOS process and finally in section 5 conclusions are given. 


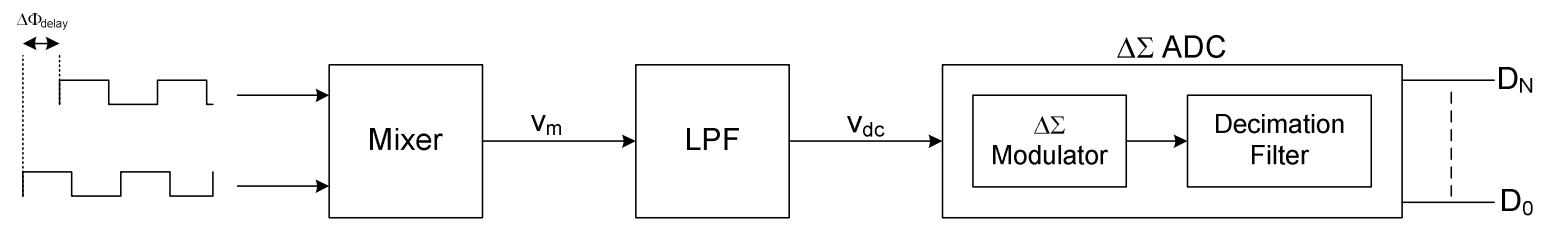

Figure 1: Proposed Time Measurement Architecture

\section{Homodyne Technique for Time Measurement}

The Homodyne technique was originally developed for RF oscillators [13]. This paper investigates this technique for producing high resolution on-chip time measurements and proposes a new fully integrated onchip time measurement architecture (Fig 1) that is based on time-to-digital conversion method. The architecture is composed of three main components: an analogue mixer, a filter and an analogue-to-digital converter (ADC). The homodyne technique converts a phase difference into a dc voltage $\left(\mathrm{V}_{\mathrm{dc}}\right)$ by modulating the input with a reference phase signal. A low pass filter is coupled to the output of the mixer to produce a $\mathrm{dc}$ voltage that is a function of the phase difference $\left(\Delta \Phi_{\text {delay }}\right)$. The modulation is achieved by the use of an analogue mixer, followed by a low pass filter. For example, if two sinusoidal signals with the same amplitude, $A$ and frequency, $\omega$, have different phases, $\phi_{1}$ and $\phi_{2}$, respectively, then the output of the mixer can be expressed as

$$
V_{m}(t)=A^{2} \cos \left(\omega t+\phi_{1}\right) \cos \left(\omega t+\phi_{2}\right)
$$

and

$$
V_{m}(t)=\frac{A^{2}}{2}\left(\cos \left(\phi_{1}-\phi_{2}\right)+\cos \left(2 \omega t+\phi_{1}+\phi_{2}\right)\right)
$$

The low pass filter is used to remove the $2 \omega$ term, leaving only the dc term which is proportional to the cosine of the phase difference multiplied by a constant.

$$
V_{d c}(t)=\frac{A^{2}}{2}\left(\cos \left(\phi_{1}-\phi_{2}\right)\right)
$$

To achieve high timing resolution the mixer circuit design needs to meet some conflicting requirements including, speed and area. Such requirements can be met using the cascode mixer circuit [14] in Figure 2. Transistors M3 and M4 form a constant current source.
Two input Vin1 and Vin2 are applied to the gates of transistors M1 and M2 respectively and the output of the mixer is taken from the drains of transistors M2 and M4. The transistor sizing was chosen to achieve the required bias currents of $10 \mu \mathrm{A}$ and appropriate driving capabilities to the filter. The cascode mixer has the additional advantage of operating with low supply voltages needed for the $1.2 \mu \mathrm{m}$ technology used for implementation. For the design of the Low Pass Filter (LPF) of the proposed architecture, a switchedcapacitor bi-quad with a cut-off frequency of $100 \mathrm{KHz}$ was used [15].

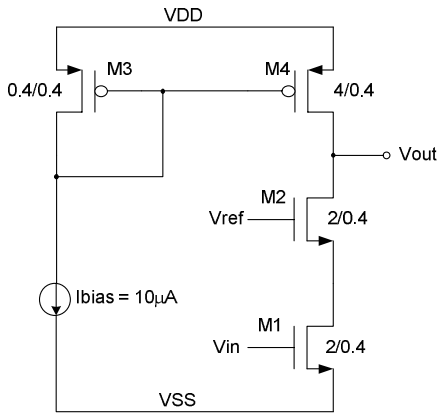

Figure 2: Cascode Mixer

\section{Analogue-to-Digital Conversion}

The purpose of the ADC is to convert the dc voltage from the output of the filter to a digital output code. The key to achieving femtosecond resolution using the proposed architecture is the implementation of a high resolution ADC. For this reason, the Delta-Sigma $(\Delta \Sigma)$ ADC was selected as opposed to Nyquist rate ADCs because of its high resolution capabilities, area requirements and also the accuracy of the converter does not depend on precise component matching, precise sample-and-hold circuitry or trimming, like Nyquist converters such as Successive Approximation and Dual Slope ADCs [16].

\section{Delta Sigma $(\Delta \Sigma)$ ADC}

The $\Delta \Sigma$ ADC depicted in Figure 1 consists of a $\Delta \Sigma$ modulator and a decimation filter. There exists 
numerous $\Delta \Sigma$ ADC architecture [17] and the choice usually involves trade-offs between resolution, circuit complexity and stability. Through extensive simulations, we have found that a $1^{\text {st }}$-order $\Delta \Sigma$ ADC with an oversampling ratio (OSR) of 32 is sufficient to achieve femtosecond resolution avoiding stability and complexity issues often associated with higher order converters. A block diagram of the $1^{\text {st }}$-order $\Delta \Sigma$ modulator is shown in Figure 3. It consists of an integrator and a single bit quantizer.

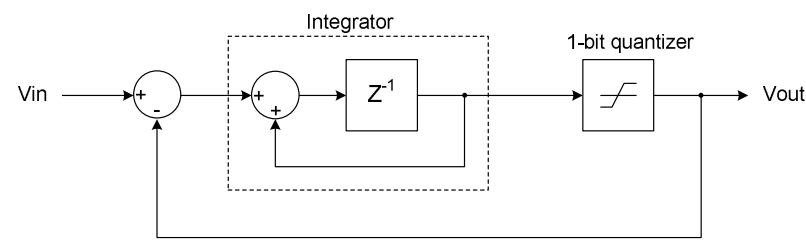

Figure 3: Block diagram of the 1st Order $\Delta \Sigma$ Modulator

The oversampling ratio (OSR) of the modulator is given by the following equation

$$
O S R=\frac{f_{s}}{2 f_{B}}
$$

Where $f_{s}$ is the sampling frequency and $f_{B}$ is the input signal bandwidth. For this application the OSR is set to 32 , in order to provide to appropriating noise shaping required to achieve the high resolution time measurement. The $\Delta \Sigma$ modulator is based on a switched-capacitor implementation and is shown in Figure 4.

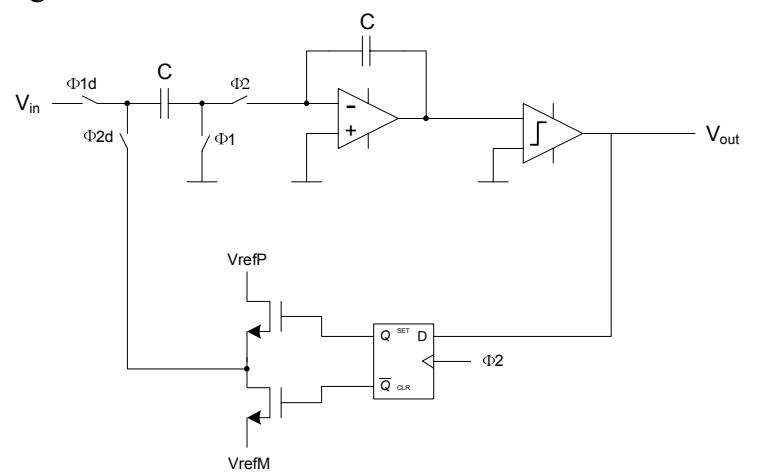

Figure 4: $1^{\text {st }}$ order switched-capacitor $\Delta \Sigma$ modulator

The $\Delta \Sigma$ modulator operates on a two phase clocking scheme, where both normal and delayed versions of the clock are generated to avoid signal dependant charge injection. The two phase clock generator is shown in Figure 5 and the clocking scheme is shown in Figure 6.

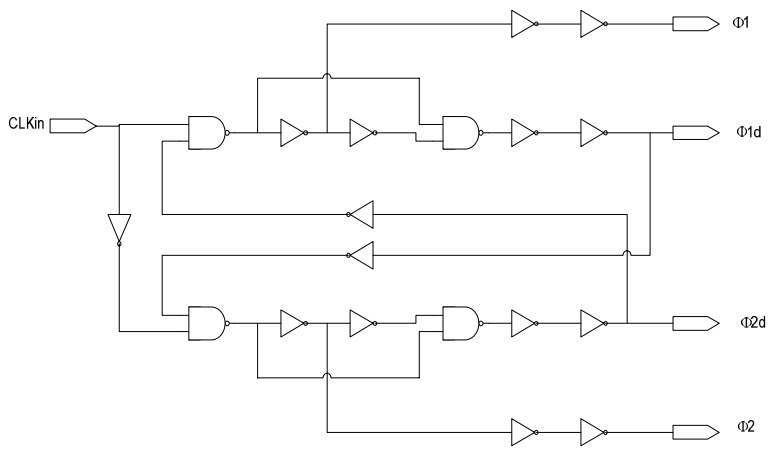

Figure 5: Non-overlapping clock generator

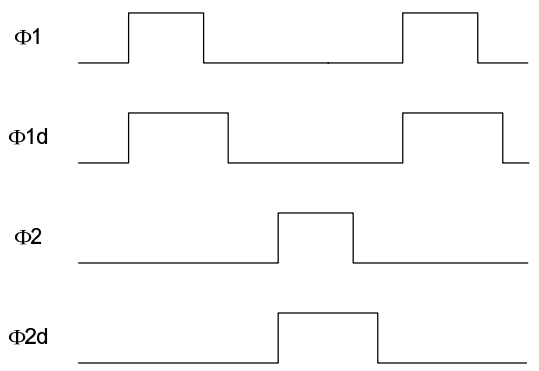

Figure 6: Non-overlapped clock timing

\section{Amplifier Design}

The amplifier employed in the integrator is a critical element within the $\Delta \Sigma$ modulator (Fig. 4). Any integrator leakage resulting from the finite dc gain of the amplifier will reduce the modulator attenuation of the quantization noise at low frequencies. Although the modulator can tolerate non-ideal components, the required dc gain of the amplifier is chosen slightly higher than the oversampling ratio. Figure 7 shows the amplifier employed in the modulator. It is based on the folded cascode operational amplifier and has a class $\mathrm{AB}$ output stage so that the output can swing close to the supply voltages. The bias circuitry is not shown for clarity. The transistor sizes in the amplifier have been designed to achieve a DC gain of $60 \mathrm{~dB}$.

\section{Comparator}

The comparator used for the single bit quantizer within the $\Delta \Sigma$ modulator (Fig 4) is shown in Figure 8. The input of the comparator consists of a rail-to-rail input stage that contains two complementary differential pairs in parallel. When one of the common mode (CM) inputs is close to VDD, differential pair M1 and M2 is active. When the CM input is close to VSS, differential 


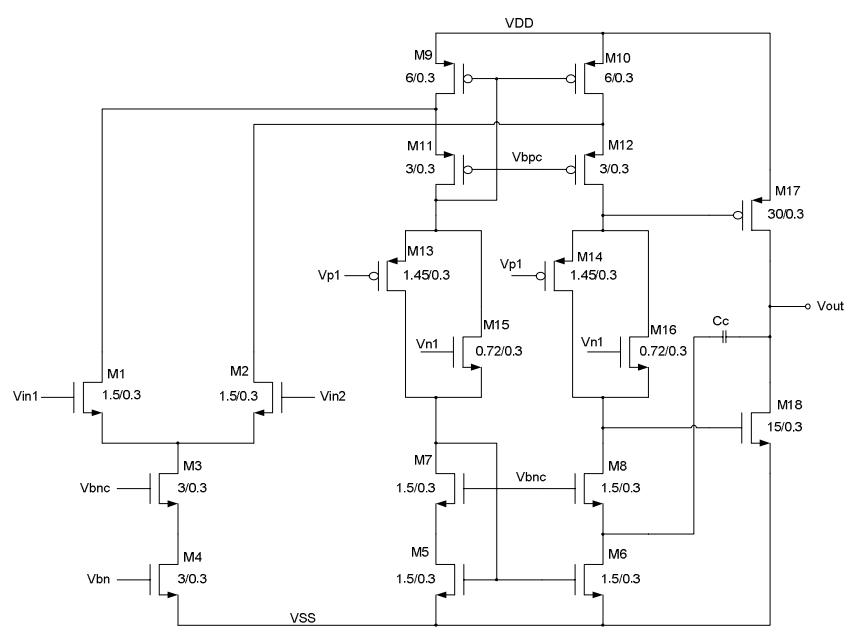

Figure 7: Folded cascode operational amplifier.

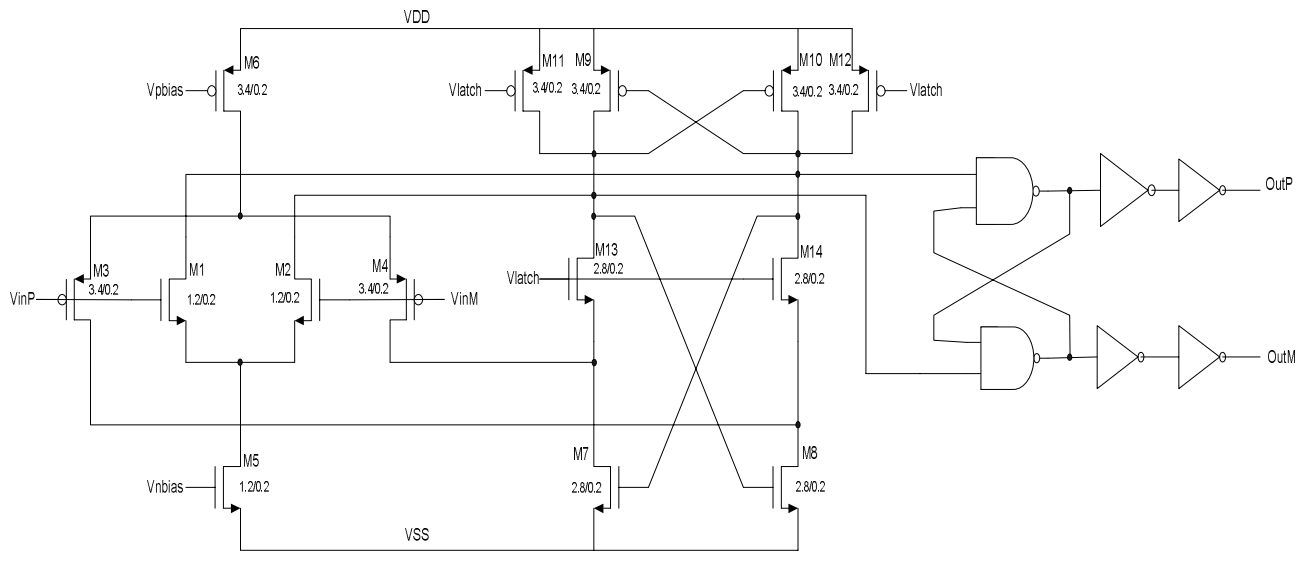

Figure 8: Comparator

pair M3 and M4 is active. Vpbias and Vnbias are the bias voltages for the tail currents of the two differential pairs which is supplied by a bias circuit; this is not shown for simplicity. The operation of the comparator is as follows. When Vlatch is low, the comparator is in a reset state and transistors M11 and M12 couple the drains of transistors M9 and M10 to VDD. Transistors M13 and M14 are off and there is no supply current flowing. When Vlatch is high, transistors M11 and M12 are open. The cross coupled regenerative inverters amplify the voltage difference and one of the output nodes is at VDD and the other is at VSS. Figure 9 shows the comparator simulation results. The comparator is clocked at $2.5 \mathrm{GHz}$ as shown by the first plot. A $500 \mathrm{MHz}$ clock signal is applied to the positive input (VinP) and the negative input (VinM) of the comparator is varied from $1.08 \mathrm{~V}(90 \%$ of VDD), $0.6 \mathrm{~V}$ with $\pm 1 \mathrm{mV}$ offset and $120 \mathrm{mV}(10 \%$ of VDD). The propagation delay of the comparator (measured from

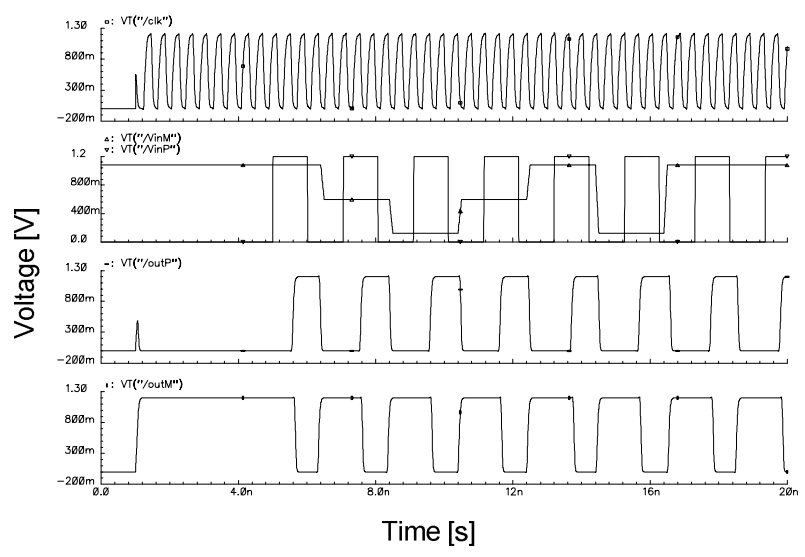

Figure 9: Comparator Simulations 
the $50 \%$ of the clock signal to $50 \%$ of the low to high transition) of $200 \mathrm{ps}$ is achieved with loading of $50 \mathrm{fF}$.

\section{Decimation Filter}

The Decimation filter of the $\Delta \Sigma$ ADC that follows the $\Delta \Sigma$ modulator is shown in Figure 10[17]. The filter performs both digital filtering and down-sampling of the single bit input data stream form the modulator. The architecture of the decimation filter consists of a counter, a clock divider and a register, making it suitable for time measurement architectures as it is small and easy to integrate. The divide-by ratio of the clock divider is set equal to the oversampling ratio (OSR) of the $\Delta \Sigma$ modulator, in this case the OSR is set to 32 .

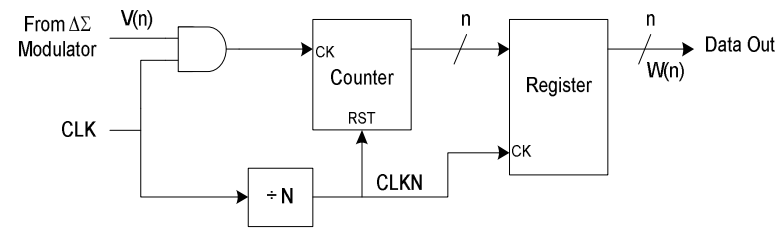

Figure 10: Decimation Filter

\section{Simulation Results}

In order to verify the performance of the proposed timing measurement architecture based on the time-todigital conversion method using the homodyne technique, the circuit level designs, considered in section 3, were implemented using a $0.12 \mu \mathrm{m}$ CMOS process. Using the SPECTRE simulator together with foundry transistor models, Figure 11 shows the output of the low pass filter of the proposed architecture when the input phase delay of two clock signals running at a frequency of $1 \mathrm{GHz}$ was varied from 0 to $500 \mathrm{fs}$. As it can be seen, there a linear relationship between the voltage output and the phase difference of the input as expected.

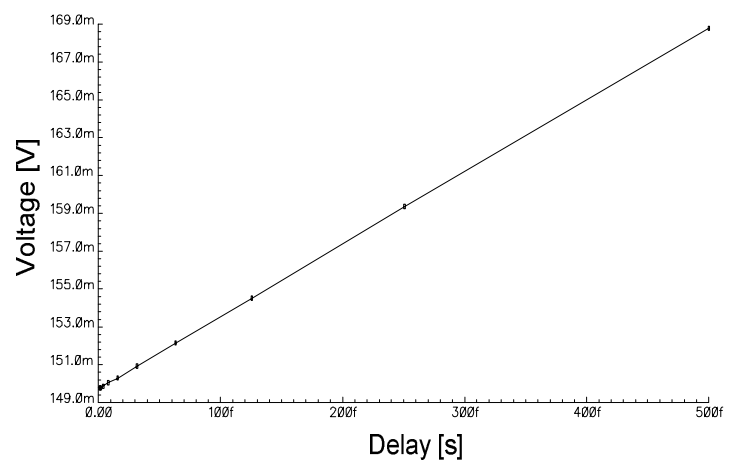

Figure 11: Simulated relationship between timing resolution and the output of the LPF.

To further demonstrate the correct operation of the proposed time measurement architecture, Figure 12 shows a simulation of two clock inputs (Clock_in and Clock_ref) with a phase difference $(\Delta \Phi)$ of $40 \mathrm{fs}$ (top plots), the mixer and filter outputs are shown in the third and forth plot, respectively and the output from the $\Delta \Sigma$ modulator is shown in the fifth plot.

To give insight into silicon area, careful layout techniques, such as common centroid layout of critical transistors (e.g. input transistors of opamp and comparator circuits) and separated analogue and digital layouts were used to reduce offsets and switching noise that would affect the overall performance of the time measurement architecture. The measurement core occupies a silicon area of $150 \mu \mathrm{m}$ by $180 \mu \mathrm{m}$, making it attractive for high resolution on-chip time measurement.

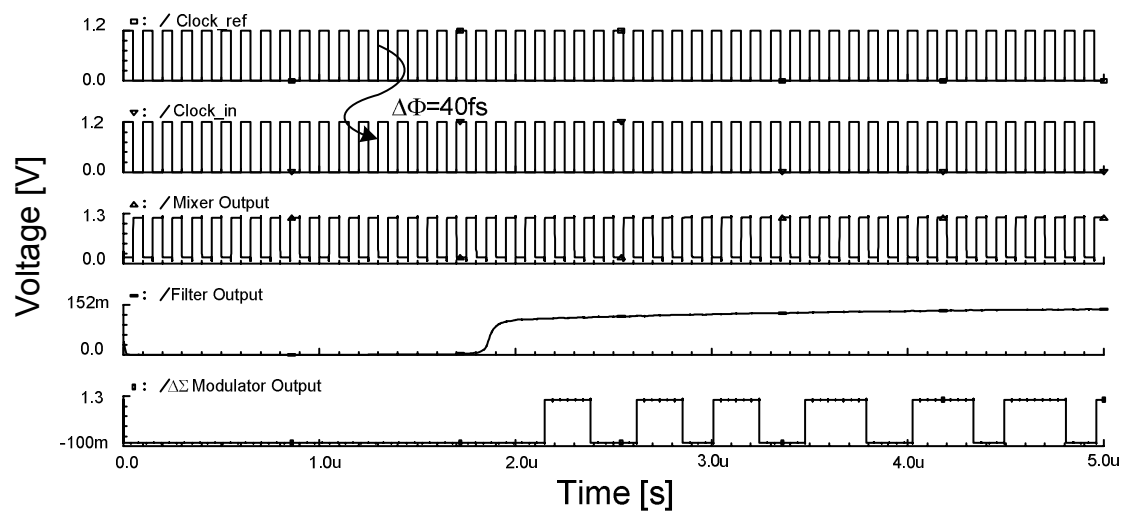

Figure 12: Input and output waveforms of the proposed architecture. 


\section{Conclusions}

This paper has investigated the capability of the Homodyne technique, which was originally developed for RF oscillators, for producing high resolution time measurements. We have proposed a new on-chip time measurement architecture based on the TDC method using the homodyne technique. It has been shown that such a technique is effective in achieving high resolution of tens of femtoseconds which is need for high performance VLSI devices operating with clock frequencies of tens of Gigahertz. This has been made possible through appropriate sections of mixer, filter and data conversion techniques. Simulations using SPECTRE models based on the $0.12 \mu \mathrm{m}$ CMOS process show that measurements are capable with a resolution of $40 \mathrm{fs}$ which is the highest reported to-date.

\section{Acknowledgement}

This work is supported in part by the EPSRC, U.K., under grant GR/S95770.

\section{References}

[1] "International Technology Roadmap for Semiconductors", http://www.itrs.net/Common/2005ITRS/Home2005.ht m, 2005.

[2] P. M. Levine and G. W. Roberts, "High-resolution flash time-to-digital conversion and calibration for system-on-chip," IEE Proceedings on Computers and Digital Techniques, vol. 152, no. 3, pp. 415-426, 2005.

[3] T. Xia and J.-C. Lo, "On-Chip Short-Time Interval Measurement for High-Speed Signal Timing Characteristics," Proceedings of the 12th Asian Test Symposium (ATS'03), pp. 326-331, 2003.

[4] S. Tabatabaei and A. Ivanov, "Embedded timing analysis: A SoC infrastructure," IEEE Design and Test of Computers, vol. 19, no. 3, pp. 22-34, 2002.

[5] T. Xia, H. Zheng, J. li, and A. Ginawi, "Self-refereed on-chip jitter measurement circuit using Vernier oscillators," IEEE Computer Society Annual Symp. on VLSI pp. 218-223, 2005.

[6] T. Xia and J.-C. Lo, "Time-to-Voltage Converter for On-Chip Jitter Measurement," IEEE Trans. on Instrumentation and Measurement, vol. 52, no. 6, pp. 1738-1748, 2003.
[7] M. Collins, B. M. Al-Hashimi, and N. Ross, "A Programmable Time Measurement Architecture for Embedded Memory Characterization," Proceedings of the 10th IEEE European Test Symposium (ETS'05), pp. 128-133, 2005.

[8] C.-C. Chen, P. Chen, C.-S. Hwang, and W. Chang, "A Precise Cyclic CMOS Time-to-Digital Converter with Low Thermal Sensitivity," IEEE Trans. on Nuclear Science, vol. 52, no. 4, pp. 834-838, 2005.

[9] P. Dudek, S. Szczpanksi, and J. V. Hatfield, "A HighResolution CMOS Time-to-Digital Converter Utilizing a Vernier Delay Line," IEEE Trans. on Solid-State Circuits, vol. 35, no. 2, pp. 240-247, 2000.

[10] E. Raisanen-Ruotsalainen, T. Rahkonen, and J. Kostamovaara, "A BiCMOS Time-to-Digital Converter with 30 ps Resolution," IEEE Inter. Symp. on Circuit and Systems (ISCAS'99), pp. 278-281, 1999.

[11] M. Oulmane and G. W. Roberts, "A CMOS time amplifier for Femto-second resolution timing measurement," Proceedings of the Inter. Symp. on Circuits and Systems (ISCAS'04), pp. 509-512, 2004.

[12] M. A. Abas, G. Russell, and D. J. Kinniment, "Design of sub-10-picosends on-chip time measurement circuit," Proceedings of the Design, Automation and Test in Europe Conference and Exhibition (DATE'04), pp. 804-809, 2004.

[13] F. O'Mahoney, C. P. Yue, M. A. Horowitz, and S. S. Wong, "A 10-GHz global clock distribution using coupled standing-wave oscillators," IEEE Journal of Solid-State Circuits, vol. 38, no. 11, pp. 1813-1820, Nov 2003.

[14] M. Madihian, H. Fujii, H. Yoshida, H. Suzuki, and T. Tamazaki, "A 1-10GHz 0.18um-CMOS Chipset for Multi-Mode Wireless Applications," IEEE Inter. Microwave Symposium (MTT-S), pp. 1865-1868, 2001.

[15] R. Gregorian and G. C. Temes, Analog MOS Integrated Circuits for Signal Processing: John Wiley and Sons, 1986, ISBN 0-471-09797-7.

[16] J. C. Candy and G. C. Temes, Oversampling DeltaSigma Data Converters: IEEE Press, 1992.

[17] R. Schreier and G. C. Temes, Understanding DeltaSigma Data Converters: John Wiley \& Sons, Inc, 2004, ISBN: 0-471-46585-2. 